\title{
Microwave Whispering-Gallery-Mode Photoconductivity Measurement of Recombination Lifetime in Silicon
}

\author{
Vladimir Yurchenko $^{1 *}$, Tugba Navruz ${ }^{1}$, Mehmet Ciydem $^{2}$, Ayhan Altintas $^{3}$ \\ ${ }^{1}$ Electrical and Electronics Engineering Department, Gazi University, Ankara, Turkey \\ ${ }^{2}$ Engitek Engineering Technologies Ltd, Ankara, Turkey \\ ${ }^{3}$ Electrical and Electronics Engineering Department, Bilkent University, Ankara, Turkey \\ *corresponding author, E-mail: v •yurchenko.nuimegmail.com
}

\begin{abstract}
We present a whispering-gallery-mode resonance-enhanced microwave-detected photoconductivity-decay method for contactless measurement of recombination lifetimes in high-resistivity semiconductor layers. We applied the method to undoped $\mathrm{Si}$ wafers of high resistivity, at 5 and 30 $\mathrm{kOhm}^{*} \mathrm{~cm}$, and measured conductivity relaxation times of 10 and 14 microseconds, respectively. In the wafers being considered, relaxation times are likely to be defined by the electron-hole diffusion from the bulk to the wafer surface.
\end{abstract}

\section{Introduction}

We present a method of resonance-enhanced contactless microwave photoconductivity measurement of the electronhole recombination lifetime $\tau_{R}$ in pure semiconductor wafers using a whispering-gallery-mode (WGM) resonator. We used the method for measuring $\tau_{R}$ in two kinds of Silicon ( $\mathrm{Si}$ ) wafers specified by the resistivity values $\rho_{1}=30$ $\mathrm{kOhm} \cdot \mathrm{cm}$ and $\rho_{2}=5 \mathrm{kOhm} \cdot \mathrm{cm}$. The samples were undoped float-zone (FZ) wafers $s_{1}$ and $s_{2}$ of diameter $D=$ $75 \mathrm{~mm}$ and thickness $d_{1}=0.5 \mathrm{~mm}$ and $d_{2}=0.4 \mathrm{~mm}$, respectively.

The method is based on the effect of resonant photosensitivity of microwave transmission through a strip-line structure loaded with a WGM resonator that has a toplayer semiconductor subject to pulsed LED illumination [1] (Figs. 1 - 3). This is one of contactless methods for electron-hole recombination measurements, which are of interest in photovoltaic technology and other areas, e.g., in the design of light-controlled millimeter-wave beam switches and similar devices [2].

The method being proposed is an enhancement of contactless methods based on the microwave-detected photoconductance-decay technique [3-9]. The methods of microwave-detected photoconductivity ( $\mu \mathrm{PCD})[9]$ and time-resolved photoluminescence (TRPL) [10] are the most powerful tools for contactless characterization of electronhole recombination in semiconductors.

The TRPL methods detect the rate of photon emission from excess carriers excited by light or other sources. They provide a direct measure of excess carrier density,

The work was presented at the URSI-TR'2018 IX Scientific Congress.

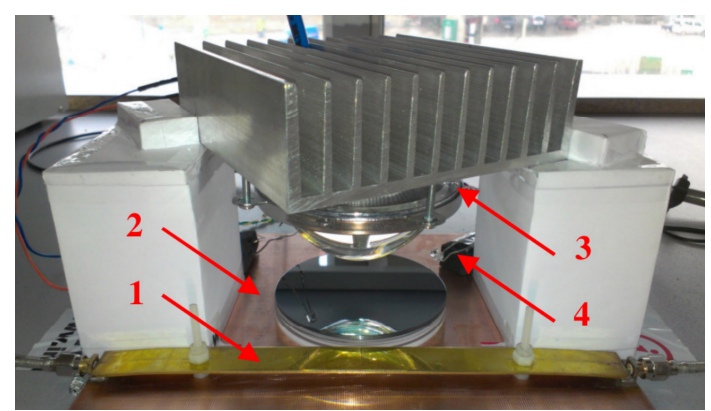

Figure 1: An experimental setup including a strip line (1), a WGM resonator (2), a heat sink with an LED and a light focusing lens (3), and a pin-diode photo-detector (4).

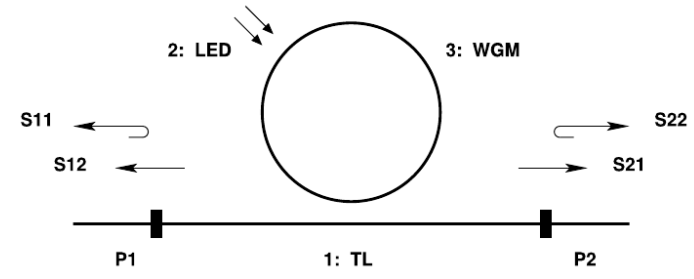

Figure 2: Schematic of a microwave WGM resonator [1].

though applied only to direct-gap semiconductors, which produce sufficient photoluminescence, e.g., GaAs. The $\mu \mathrm{PCD}$ methods measure the conductivity induced by light, which is proportional to the product of density and mobility of charge carriers. They can be used with both directgap and indirect-gap semiconductors, e.g., Si [5-7]. Both the techniques, being contactless methods, have essential benefits over other approaches, though they require highpower laser sources for creating significant photo-excitation and, typically, are implemented using expensive equipment made for industrial applications [4].

For advancing $\mu \mathrm{PCD}$ methods, an enhancement of the photoresponse was proposed based on the use of resonant circuits and structures. This resulted in the development of resonance coupled [8] and transmission modulated [9] photoconductive decay techniques, RCPCD and TMPCD, respectively, which showed a big improvement in the recombination measurements despite the fact that the quality factor $Q$ of the resonators was rather limited. 
There are different mechanisms of electron-hole recombination, which may coexist, dominate, or replace one another, depending on the kind of semiconductor (direct- or indirect-gap), kind of doping ( $\mathrm{p}$ - or n-type, degenerate or not, doped, undoped, or compensated), level of excitation (low or high) and other conditions [10]. The most common are Shockley-Read-Hall recombination via impurity centers, radiative inter-band transitions, and Auger impact recombination, which are characterized by different dependencies on the electron-hole densities and different sets of recombination parameters.

In a broad range of circumstances, a generic concept of recombination lifetime can be introduced that corresponds to the exponential decay of carrier excitation in a semiconductor [11]. In practice, the relaxation may reveal a few exponential terms or show non-exponential decay. The relaxation is more complicated when surface recombination is involved or barrier structures exist where carrier transport is essential. We consider undoped uniform semiconductor samples of relatively small thickness $(\sim 0.5 \mathrm{~mm})$ with no special surface treatment and barrier structures, though assuming a possibility of surface recombination, which is a common property of Silicon samples [5-7].

Typically, it is difficult to measure recombination parameters at low excitation level when the carrier density is on the order of $10^{15}$ carriers $/ \mathrm{cm}^{3}$, since the signal is low and noisy, and the dynamic range is limited $[5,10]$. So, most of the measurements for common semiconductors were made under high excitation conditions that correspond to $10^{16}$ $10^{17}$ carriers $/ \mathrm{cm}^{3}$ and above. At the same time, the lowexcitation limit is of interest, since the recombination lifetime is sensitive to the injection level. This is also the kind of condition that occurred in our experiments in $[1,2]$.

Despite the abundance of measurement techniques, we could not find any contactless method that would be suitable for our Si wafers in a range of accessible laboratories around. In the meantime, trials of a more conventional technique (pressed-contact photoconductance) failed due to uncontrollable nonlinear effects of electron transport through unpredictable contact barriers and in the bulk of high-resistivity Si wafers. Besides, measurements of this kind require strong illumination that creates further problems with this approach.

These circumstances created a stimulus for the development of a contactless measurement method that could be implemented in a laboratory using available technical means and equipment.

The aim of this work is to develop a resonanceenhanced WGM-based microwave photoconductivity method for contactless measurement of recombination lifetime in pure semiconductors and to apply it to the measurement of recombination lifetime in high-resistivity Si wafers. The advantage of the technique is the possibility of making measurements at low light intensity and low level of excitation of the semiconductor.

A novelty of the approach is the use of microwave WGM resonators with high quality factors [12], which

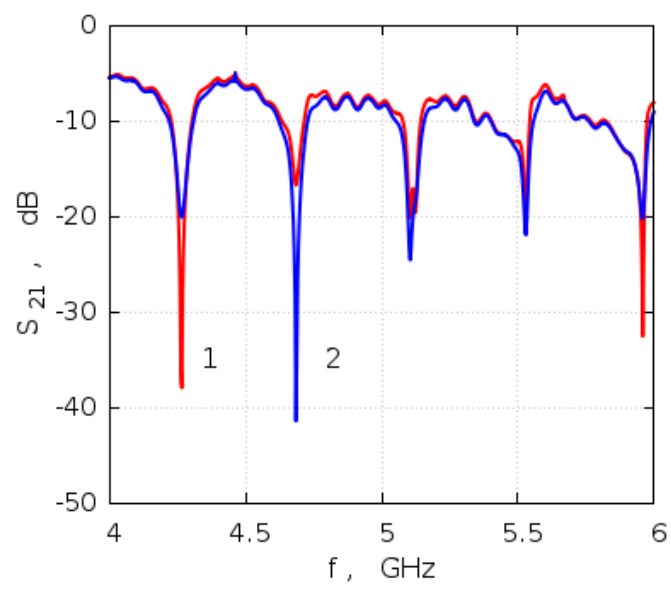

Figure 3: Transmission spectrum of a microwave WGM setup in the dark (curve 1) and in the light (curve 2).

greatly increases the sensitivity of a system that is based on conventional laboratory equipment. Should ultra-fast sub$\mathrm{THz}$ Schottky barrier diodes be employed as millimeterwave signal detectors [13], Si-loaded W-band Bragg resonators [2] with greater $Q$ factors at higher frequencies could be used for the detection of faster recombination processes producing much lower photoresponse.

\section{Setup of the Whispering-Gallery-Mode Photoconductivity-Decay Method}

The method is based on the use of devices and components available in a typical RF laboratory. The main device is the R\&S FSH8 Handheld Spectrum Analyzer (model .28 with FSH-K42 option) that operates as a vector network analyzer (VNA) in the frequency band $f=100 \mathrm{kHz}-$ $8 \mathrm{GHz}$ with a possibility of time-domain signal tracking at the chosen frequency (the best time resolution in this tracking is $\Delta t=1 \mu \mathrm{s}$ ). High-resistivity Silicon wafers, depending on their intended applications, may have the recombination lifetime $\tau_{R}$ ranging from nearly a second (ultra-pure samples) to nano- and picosecond scales (radiation damaged wafers). For typical undoped Silicon, $\tau_{R}$ is likely to be on a millisecond to microsecond scale that matches the time resolution of the VNA specified above.

The method utilizes the effect of resonant photosensitivity of microwave transmission through a strip line coupled to the disk WGM resonator that contains a semiconductor layer [1]. The measurement system (Figs. 1, 2) consists of (1) a strip line suspended over a conducting plate, (2) a WGM resonator made of a set of dielectric and semiconductor wafers placed on the same plate, (3) a 100W blue or white LED array with a lens attached to the heat sink, and (4) a pair of pin-diode photo-detectors for measuring the light intensity, which is controlled by the LED array voltage $V_{L E D}$.

In order to operate at frequencies below $8 \mathrm{GHz}$, the resonator in this setup contains more dielectric wafers as compared to the WGM structure in [1]. The entire WGM 
resonator consists of $7 \mathrm{MgF}_{2}$ wafers placed on the copper conducting plate, 9 sapphire wafers layed above (wafers of thickness $0.55 \mathrm{~mm}$ and $0.40 \mathrm{~mm}$, respectively), and a top stack of a few Si wafers facing the LED. The top stacks were of the kinds: $3 s_{1}-2 s_{2}$-LED (\#1), $2 s_{2}-3 s_{1}$-LED (\#2), $1 s_{1}$-LED (\#3), and $1 s_{2}$-LED (\#4), where $s_{1}$ and $s_{2}$ are the Si wafers specified above (alternatively, the entire resonator can be assembled from high-resistivity $\mathrm{Si}$ wafers).

The LED is controlled by an electronic switch providing a fast $(<1 \mu \mathrm{s})$ switching off the light pulse (faster than $0.2 \mu$ s for the blue LED) and generating a synchronized trigger signal for the VNA operation. The strip line is connected to the VNA ports so that the microwave transmission signal is measured as a function of either the frequency $f$ (over the given band at the fixed light intensity, Fig. 3) or the time $t$ (at the chosen microwave frequency, when synchronized with an event of turning off the light pulse, Fig. 4).

\section{WGM Enhanced Measurements of the Photoconductivity Decay Times}

Fig. 3 shows two kinds of resonances observed as the narrow dips in $S_{21}$ that emerge either (1) in the dark state or (2) under the light of the blue LED (the peak values of resonator quality factors are $Q \sim 10^{4}$, unlike $Q \sim 20$ in another technique [3], but the resonator lifetime is still reasonably small, with an instant peak value being $<1.6 \mu \mathrm{s}$ ). When setting the frequency in the time-domain measurements at the resonant point, we observe either the downswitch or the up-switch dynamics of $S_{21}$ signals, respectively (see Fig. 4, a and b). Some dips may have their minimum at a medium light intensity. Then, we observe a hybrid switch (Fig. 4, c) that corresponds to a system of twice greater sensitivity, thus improving the detection of small carrier density variations.

Figs. 5 - 7 show three kinds of result, which represent the main stages of the measurement process. The first result (Fig. 5) shows the transmission spectra $S_{21}(f)$ of setup \#3 as measured at different light intensities, which are related to cases (b) and (c) in Fig. 4.

The second result (Fig. 6) is the calibration curve $V_{R}\left(S_{21}\right)$ recovered from these spectra. The curve provides the relationship between the light intensity presented by the photo-detector signal $V_{R}$ and the microwave transmission signal $S_{21}$, which is recorded under exactly the same conditions (including the same frequency $f_{0}=6.231000 \mathrm{GHz}$ ) as used in the time-domain measurements of $S_{21}$ in Fig. 4, $\mathrm{b}$ and $\mathrm{c}$.

The third, and the main result (Fig. 7), is the actual timedomain curve $V_{R}(t)$ showing the recombination dynamics of the photo-excited charge carriers (the effective photoconductivity, whose value is proportional to the $V_{R}$ light signal, i.e., the excess carrier density). The $V_{R}(t)$ signal is recovered from the VNA measured $S_{21}(t)$ time-domain curve by using the calibration function $V_{R}\left(S_{21}\right)$ established above.

The $S_{21}(t)$ curve used in this procedure is found as an average of 12 synchronized time-domain up-switch traces

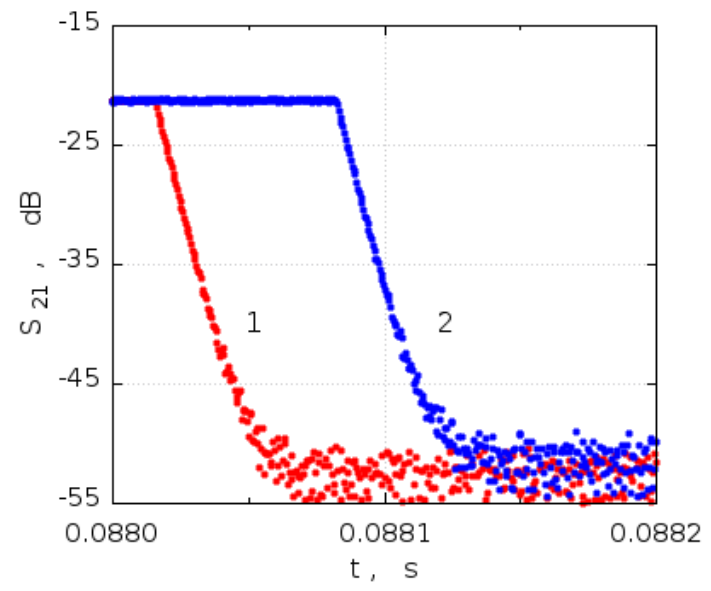

(a)

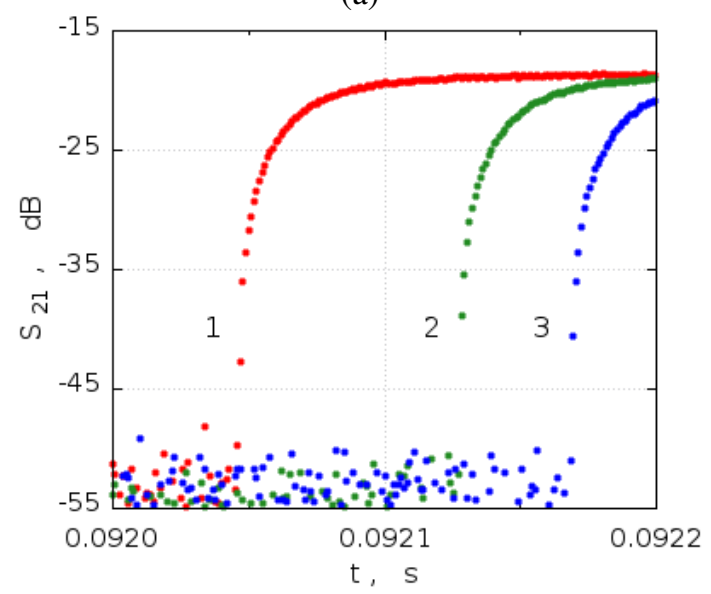

(b)

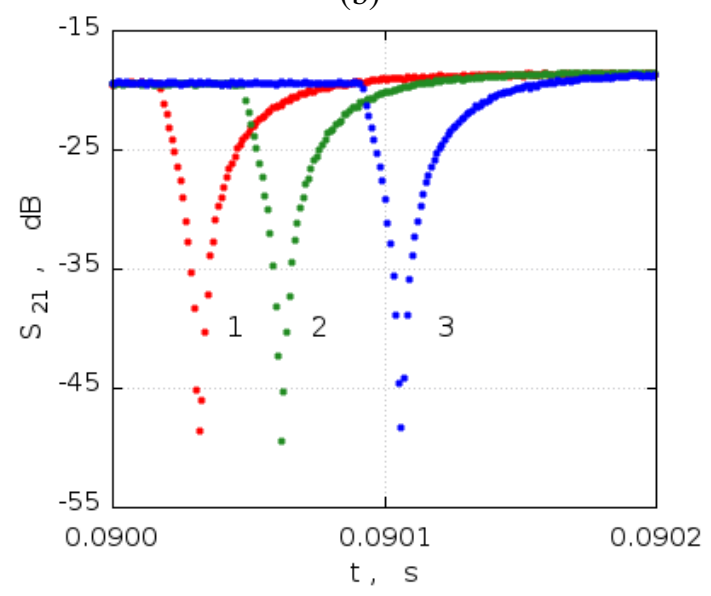

(c)

Figure 4: VNA signal measured in the time-domain at the resonant frequency $f_{0}$ when turning off the light in the case of (a) down, (b) up, and (c) hybrid switch in a setup with (a) WGM structure \#1 $\left(f_{0}=4.268882 \mathrm{GHz}, V_{L E D}=32 \mathrm{~V}\right)$ and $(\mathrm{b}, \mathrm{c})$ WGM structure \#3 $\left(f_{0}=6.231000 \mathrm{GHz}, V_{L E D}\right.$ $=30.8 \mathrm{~V}$ and $40.2 \mathrm{~V}$, respectively).

(Fig. 8) like those shown in Fig. 4, b. The traces were recorded repeatedly with sufficient intervals of dark-state relaxation between the light pulses. Fig. 8 illustrates the 


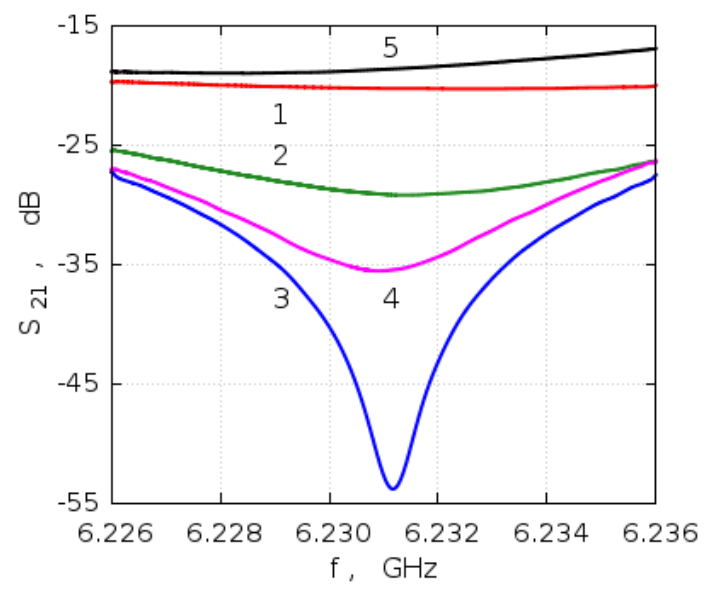

Figure 5: Transmission spectra of setup \#3 at different light intensities $\left(V_{L E D}=39,33,31,30,20 \mathrm{~V}\right.$, curves 1 to 5 , respectively).

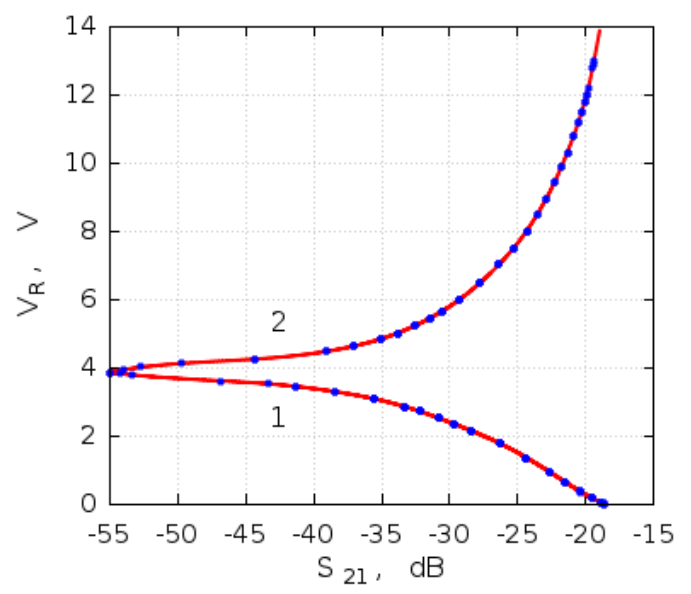

Figure 6: Calibration curve recovered from the data in Fig. 5 (branches 1 and 2 correspond to weak and strong light, respectively, dots show the measured values, and the curve is the smooth fit needed for data processing).

benefit of this averaging for computing more accurate representative curve $S_{21}(t)$ for further processing.

\section{Simulations of the Photoconductivity Decay in Semiconductor Wafers}

Recovery of recombination parameters of Si wafers from the experimental data requires numerical simulations of the relaxation dynamics of photo-excited charge carriers after the turning off the optical excitation. The electron-hole dynamics in Si wafers under the given conditions can be computed by using the drift-diffusion model of charge transfer in semiconductors under illumination [14].

In the case of high-resistivity Si wafers, we have to use the non-stationary electron-hole ambipolar diffusion equation presented in the form

$$
\frac{\partial n}{\partial t}-D_{a} \frac{\partial^{2} n}{\partial x^{2}}+\frac{n-n_{0}}{\tau_{R}}=G(x, t)
$$

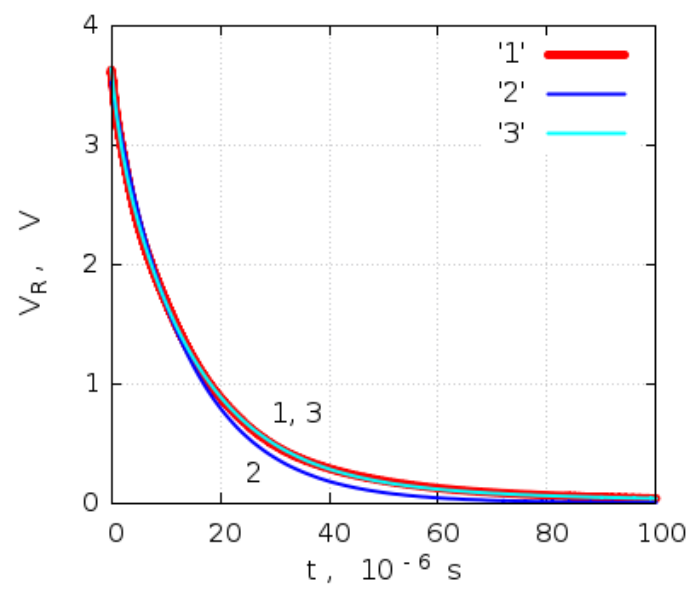

Figure 7: Time curves showing the recombination dynamics of photo-excited carriers (whose density is proportional to the light flux presented by the $V_{R}$ photo-detector signal) as recovered from the measured data (curve 1) and fitted with one-term and three-term exponential functions (curves 2 and 3, respectively), which show the dominant relaxation time $\tau_{V_{R}}=14 \mu \mathrm{s}$ (in case of $s_{2}$ wafers, $\tau_{V_{R}}=10 \mu \mathrm{s}$ ).

where $n=n_{0}+\Delta n$ is the electron density in a semiconductor under illumination, $n_{0}$ is the dark-state equilibrium electron density, $\Delta n$ is the excess density of nonequilibrium electrons created by photo-excitation, $D_{a}=$ $2 D_{n} D_{p} /\left(D_{n}+D_{p}\right)$ is the ambipolar diffusion coefficient, $D_{n}$ and $D_{p}$ are the electron and hole diffusion coefficients, $\tau_{R}$ is the electron-hole recombination lifetime, and $G(x, t)$ is the term describing the generation of the electron-hole pairs by the light flux. The wafer of thickness $d$ is located at $0 \leq x \leq d$. The light is incident on the wafer surface $x=0$ while propagating in the positive $x$ direction, which is normal to the wafer surface.

The generation term $G(x, t)$ is computed as

$$
G(x, t)=g(t) \int l^{-1} q(1-r) \exp (-x / l) d \lambda
$$

where $l=l(\lambda)$ is the length of light absorption in $\mathrm{Si}$ as a function of the wavelength $\lambda[15], q=q(\lambda)$ is the quantum flux of light at the total power flux $P_{L}, r=r(\lambda)$ is the reflection coefficient of light at the wafer surface, and $g(t)$ is the function of time specifying the dynamics of photoexcitation.

The integral is taken over the range of wavelengths specific for the given light source. We consider blue and warmwhite LEDs radiating light at the wavelengths $\lambda \sim 450 \mathrm{~nm}$ $(l \sim 0.4 \mu \mathrm{m})$ and $\lambda \sim 440-700 \mathrm{~nm}(l \sim 0.4-5.3 \mu \mathrm{m})$, respectively.

The function of time $g(t)$ is chosen to define the light pulse of duration $t_{P}$, which is turned on at $t=-t_{P}$ and turned off abruptly at $t=0$. The function is defined as $g(t)=\Theta\left(t+t_{P}\right)-\Theta(t)$ where $\Theta(t)$ is the unit step function.

The initial condition, imposed at $t=-t_{P}$, is the darkstate equilibrium condition in a semiconductor $\left(n=n_{0}\right)$. 


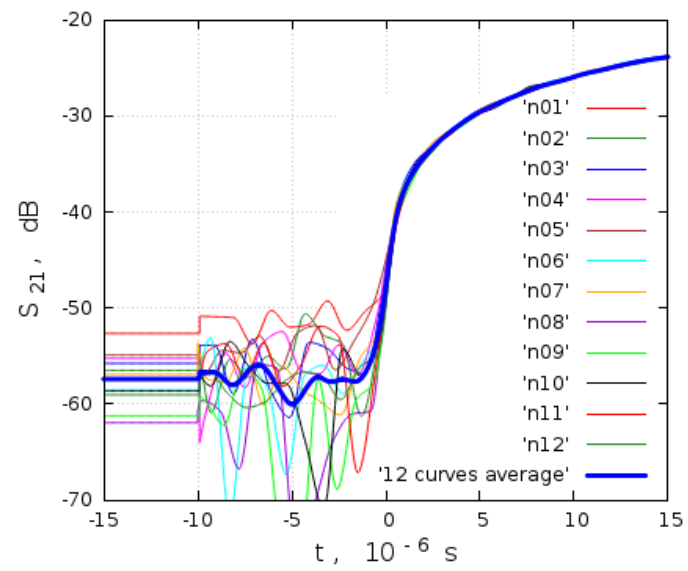

(a)

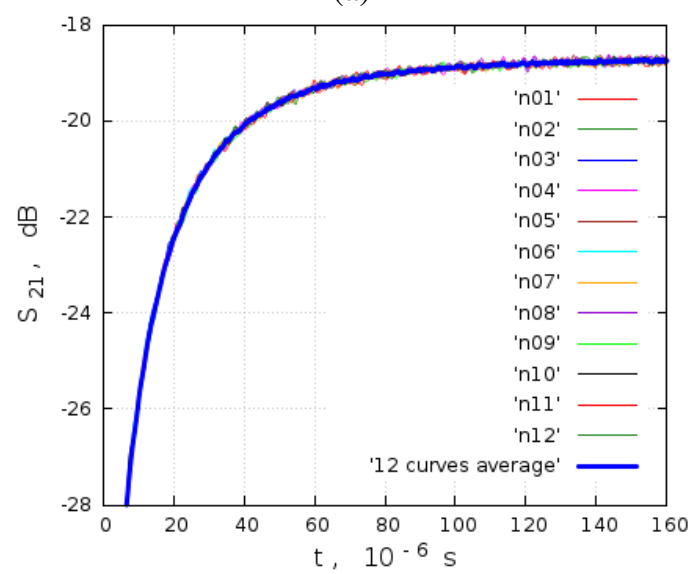

(b)

Figure 8: The $S_{21}(t)$ curve defined as an average of 12 synchronized up-switch traces when (a) $-15 \mu \mathrm{s} \leq t \leq 15 \mu \mathrm{s}$ and (b) $5 \mu \mathrm{s} \leq t<160 \mu \mathrm{s}$.

Choosing the pulse duration $t_{P} \geq 100 \mu \mathrm{s}$, we achieve a steady-state non-equilibrium carrier distribution established before $t=0$ when the light is turned off and the simulation of photoconductivity decay started.

The boundary conditions at the wafer surfaces assume the finite speed $S$ of surface recombination of charge carries. In practice, typically, the surface recombination is so high that nearly equilibrium carrier density is observed at the surfaces ( $n=n_{0}$ at $x=0$ and $x=d$ ).

The values of $D_{a}$ and $\tau_{R}$ define the electron-hole ambipolar diffusion length $L_{R}=\sqrt{D_{a} \tau_{R}}$ whose value controls the distribution of photo-excited charge carries inside the wafers. At the given wafer thickness $d$, large values of $L_{R} \quad\left(L_{R} \gg d\right)$ lead to significant redistribution of photo-excited carriers across the wafer whereas a small $L_{R}$ $\left(L_{R} \ll d\right)$ supports the carrier profile as generated by the local absorption of light.

Numerical solutions of Eqn. (1) were found for $\mathrm{Si}$ wafers for various values of the recombination parameters by using the FlexPDE simulation software. Simulation results concerning the spatial distribution of photo-electrons in a $\mathrm{Si}$ wafer and the time decay curves of the total number

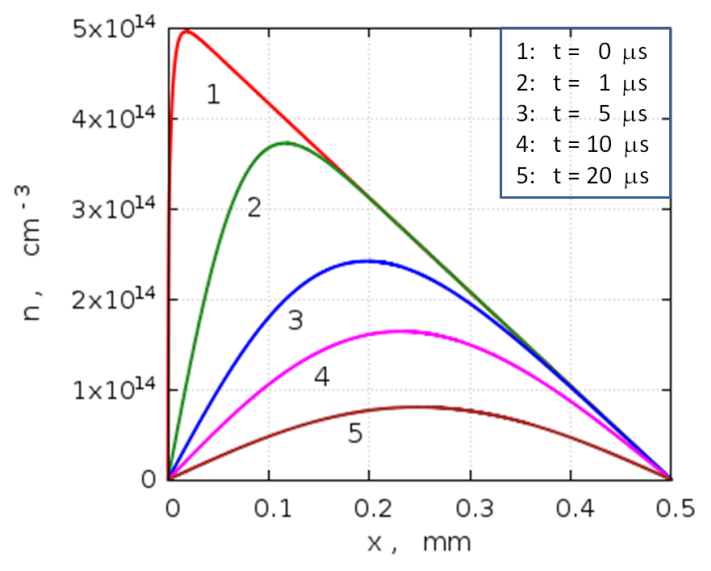

Figure 9: Decay of the photo-electron distribution in a $\mathrm{Si}$ wafer $s_{1}$ after the turning off the blue light with power flux $P_{L}=10 \mathrm{~W} / \mathrm{cm}^{2}$ at the moment $t=0$ (curves 1 to 5 show simulation results at the time $t=0,1,5,10$, and $20 \mu \mathrm{s}$, respectively, when $\tau_{R}=6000 \mu$ s and $\tau_{S}=0$ ).

of electrons per unit area $n_{\text {tot }}(t)$ after the turning off the light are presented in Figs. $9-11$.

The quantity $n_{\text {tot }}(t)=\int_{0}^{d} n(x, t) d x$ defines the effective photo-conductivity of the wafer $\sigma_{p h}(t)$ as $\sigma_{p h}(t)=$ $e\left(\mu_{n}+\mu_{p}\right) n_{t o t}(t) / d$ where $\mu_{n(p)}$ is the electron (hole) mobility and $e$ is the elementary charge.

Fig. 9 shows the time decay of the photo-electron distribution in the Si wafer $s_{1}$ after the turning off the light emitted by the blue LED with the initial power flux $P_{L}=$ $10 \mathrm{~W} / \mathrm{cm}^{2}$. The results illustrate the process of redistribution of charge carriers across the wafer along with the simultaneous decay of carrier density in the sample that leads to the decay of total conductivity as detected in microwave experiments.

It is the comparison of these simulation results with relevant experimental data that allows one to extract the recombination parameters of wafers from a set of timedomain microwave photoconductivity experiments.

\section{Extraction of the Recombination Lifetime Parameters}

For the comparison of simulated relaxation dynamics of charge carriers in a Si wafer and experimental $V_{R}(t)$ photoconductivity signal, we computed time decay curves $n_{t o t}(t)$ in a $\mathrm{Si}$ wafer for various values of recombination parameters. Then, by selecting the cases of the best fit between the measured and simulated curves, we identify those values that allow us to better reproduce the experimental data $V_{R}(t)$.

Figs. 10 and 11 show some of $n_{\text {tot }}(t)$ curves found at different values of the bulk and surface recombination times $\tau_{R}$ and $\tau_{S}$, respectively, in a Si wafer of thickness $d=0.5 \mathrm{~mm}$ (the wafer $s_{1}$ ). The curves are presented in relative units (curves 2 to 4 ) along with similarly normalized $V_{R}(t)$ experimental curves obtained above (curves 1 in 
Figs. 7, 10, and 11).

Given the surface recombination speed $S$, the surface recombination time and length are defined as $\tau_{S}=D_{a} / S^{2}$ and $L_{S}=D_{a} / S$, respectively, where $D_{a}=18 \mathrm{~cm}^{2} / \mathrm{s}$ [14] $\left(L_{S}=\sqrt{D_{a} \tau_{S}}\right)$. Then, the time parameters $\tau_{R}=20,666$, $6000 \mu$ s and $\tau_{S}=0.02,0.056,5.6,35 \mu$ s that have been tested in various combinations as possible parameter values (Figs. 9 - 11) correspond to the length parameters $L_{R}=$ $0.19,1.1,3.3 \mathrm{~mm}$ and $L_{S}=0.006,0.01,0.1,0.25 \mathrm{~mm}$, respectively.

For a quantitative analysis of time decay functions and comparison of simulation results with experimental data, we apply three-term exponential fitting of measured and simulated curves using the fitting function

$$
\eta(t)=\sum_{k=1}^{3} \eta_{k} \exp \left(-t / \tau_{k}\right)
$$

where $\eta_{k}$ is the contribution factor and $\tau_{k}$ is the relaxation time of each term. The main term is the one that has the greatest contribution factor $\eta_{k}$ that, in turn, defines the main relaxation time $\tau_{k}$.

By fitting our data, we obtain the main relaxation times $\tau_{V_{R}}=14.4 \mu \mathrm{s}, \tau_{10.2}=\tau_{11.2}=14.5 \mu \mathrm{s}, \tau_{10.3}=26 \mu \mathrm{s}$, $\tau_{10.4}=8.6 \mu \mathrm{s}$, and $\tau_{11.3}=14.0 \mu \mathrm{s}$ where the index $V_{R}$ and the numerical indices $\alpha=10.2,11.2, \ldots$, correspond to the experimental function $V_{R}(t)$ and simulated curves in Figs. 10 and 11, respectively.

Indices $\alpha=10.2,10.3$, and 10.4 specify curves 2,3 , and 4 in Fig. 10. The curves are computed with the values of $\tau_{R}=666 \mu \mathrm{s}$ (curves 2 and 3) and $\tau_{R}=20 \mu \mathrm{s}$ (curve 4) when $\tau_{S}=0.02 \mu \mathrm{s}, \tau_{S}=5.6 \mu \mathrm{s}$, and $\tau_{S}=0.056 \mu \mathrm{s}$ (curves 2, 3, and 4, respectively).

Indices $\alpha=11.2$ and 11.3 specify curves 2 and 3 in Fig. 11. These curves are obtained with the recombination parameters $\tau_{R}=666 \mu \mathrm{s}, \tau_{S}=0.02 \mu \mathrm{s}$ and $\tau_{R}=20 \mu \mathrm{s}$, $\tau_{S}=35 \mu \mathrm{s}$, respectively.

The main fitting terms have sufficiently large contribution factors $\eta_{V_{R}}=0.84, \quad \eta_{10.2}=\eta_{11.2}=0.83$, $\eta_{10.3}=0.95, \eta_{10.4}=0.77$, and $\eta_{11.3}=0.96$.

The pairs of $\tau_{R}$ and $\tau_{S}$ values used above constitute the parameter sets $P_{\alpha}$ being tested. The pairs providing a good match of simulated and experimental curves specify possible values of recombination parameters being recovered.

The results presented above show the possibility of extracting the recombination parameters of a $\mathrm{Si}$ wafer from the comparison of relaxation curves of measured and simulated photo-excitation in a semiconductor after the turning off the optical excitation.

The processing of experimental photoconductivity data reveals the main relaxation time to be $\tau_{V_{R}}=14.4 \mu \mathrm{s}$ with the contribution factor of the relevant term $\eta_{V_{R}}=0.84$. The other terms are specified by parameters $\tau_{2}=1.7 \mu \mathrm{s}$ and $\tau_{3}=59 \mu$ s at $\eta_{2}=0.11$ and $\eta_{3}=0.05$, respectively.

Simulations are capable of reproducing nearly the same value $\tau_{11.2}=14.5 \mu$ s (curve 2 in Fig. 11) at the recombination time parameters $\tau_{R}=666 \mu \mathrm{s}$ and $\tau_{S}=0.02 \mu \mathrm{s}$ ( $S=3 \cdot 10^{4} \mathrm{~cm} / \mathrm{s}$ ), which specify the bulk and the surface

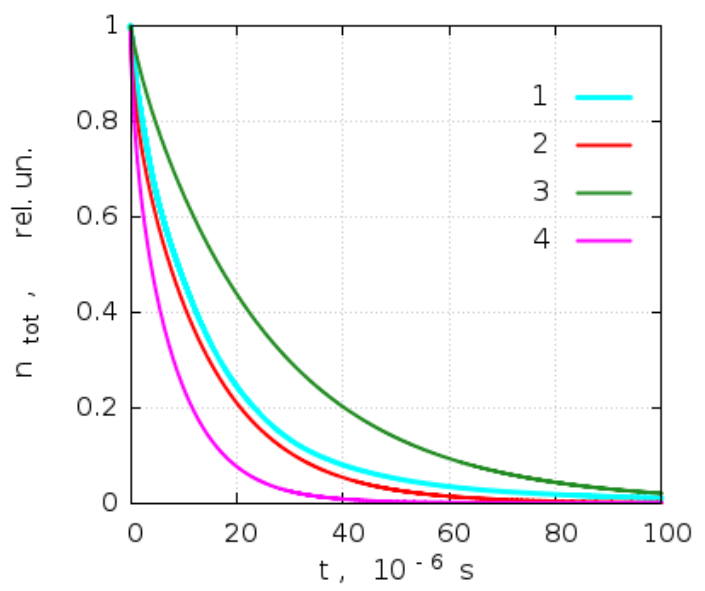

Figure 10: Decay of the total number of electrons in Si wafer $s_{1}$ after the turning off the blue light at $t=0$ where curve 1 presents experimental data and curves 2 to 4 show simulation results obtained at $\tau_{R}=666 \mu$ s (curves 2 and 3) and $\tau_{R}=20 \mu \mathrm{s}$ (curve 4) when $\tau_{S}=0.02 \mu \mathrm{s}, \tau_{S}=5.6 \mu \mathrm{s}$, and $\tau_{S}=0.056 \mu$ s (curves 2,3 , and 4 , respectively).

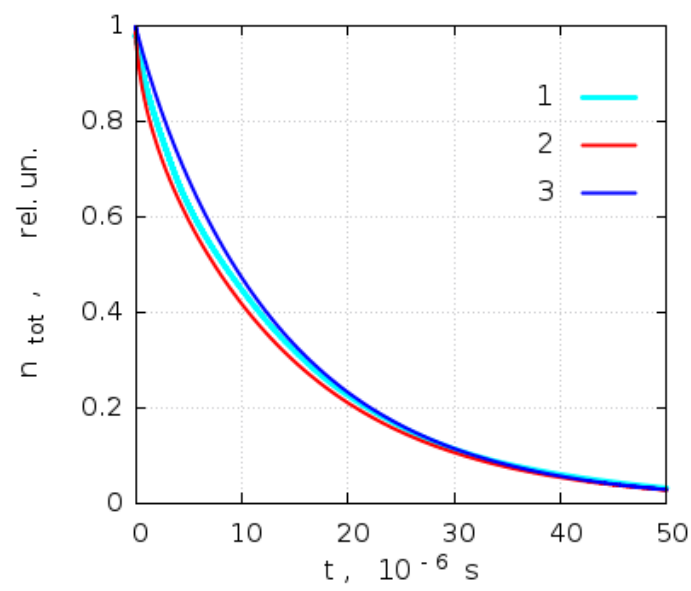

Figure 11: Decay of the total number of electrons in $\mathrm{Si}$ wafer $s_{1}$ after the turning off the blue light at $t=0$ where curve 1 shows experimental data and curves 2 and 3 show simulation results at $\tau_{R}=666 \mu \mathrm{s}, \tau_{S}=0.02 \mu \mathrm{s}$ and $\tau_{R}=20 \mu \mathrm{s}, \tau_{S}=35 \mu \mathrm{s}$, respectively.

processes, respectively. Notice, however, that values close to the same relaxation time can be obtained (though, with a greater mismatch of curves) by using quite different recombination parameters, e.g., $\tau_{11.3}=14.0 \mu$ s with $\tau_{R}=20 \mu \mathrm{s}$ and $\tau_{S}=35 \mu \mathrm{s}$.

The reason for such a situation is that, at the given thickness of a Si wafer $d$, the charge carrier diffusion from the bulk to the wafer surfaces occurs at about the same time as measured in the experiment. At the initial carrier distribution presented in Fig. 9, this time is found, approximately, as $\tau_{D} \approx(d / 3)^{2} / D_{a} \approx 15 \mu \mathrm{s}$. In case of $\tau_{S}<\tau_{D}<\tau_{R}$, it is the time $\tau_{D}$ that defines the relaxation time detected in the experiment. About the same relaxation time is also obtained when $\tau_{S} \sim \tau_{R} \sim \tau_{D}$. In this case, the process is 
controlled by all the time parameters being involved. Removing this ambiguity requires either much thicker wafers or significant suppression of surface recombination.

In practice, undoped $\mathrm{Si}$ is, usually, characterized by a large recombination lifetime, e.g., $\tau_{R}=666 \mu$ s as measured in the float-zone Si wafers [8]. At the same time, the surface recombination in $\mathrm{Si}$ is, usually, very fast, if no special treatments applied. The FZ Si wafers in our experiments are of this kind. In this case, the measured relaxation time $\tau_{V_{R}}$ is the effective quantity representing the entire sample rather than the material parameter. Nonetheless, the relaxation time $\tau_{V_{R}}$ is valuable as an engineering design parameter.

\section{Conclusions}

A resonant microwave-detection photoconductivity-decay method has been proposed for contactless recombination lifetime measurements in semiconductor wafers. The method utilizes microwave whispering-gallery-mode disk resonator structures, which can provide large quality factors $Q \sim 10^{4}$ for resonances and, therefore, a large enhancement of the sensitivity of the measurement system.

The method allows one to measure recombination lifetimes $\tau_{R}$ at a very low level of excitation $\left(\Delta n<10^{15} \mathrm{~cm}^{-3}\right.$ at $P_{L}<10 \mathrm{~W} / \mathrm{cm}^{2}$ ) and use inexpensive LED arrays as the light sources. The method has been used for measuring recombination parameters in high-resistivity floatzone Si wafers $(\rho \sim 5-30 \mathrm{kOhm} \cdot \mathrm{cm})$ with wafer thickness $d \sim 0.4-0.5 \mathrm{~mm}$, which show the relaxation times $\tau \sim 10-14 \mu \mathrm{s}$, respectively.

In wafers of small thickness under the typical condition of slow bulk and rapid surface recombination, the measured relaxation time is defined by the electron-hole diffusion to wafer surfaces rather than the bulk recombination rate.

When using wafers of greater thickness or reducing the rate of surface recombination, one can measure the bulk recombination lifetime directly, with high sensitivity, and in a broad range of light intensities, which is hard to achieve with other techniques.

\section{Acknowledgement}

The work was supported by The Scientific and Technological Research Council of Turkey (TUBITAK) through the 2221 Fellowship Program for Visiting Scientists and Scientists on Sabbatical Leave (2017/2).

\section{References}

[1] V. Yurchenko, M. Ciydem, A. Altintas, Lightcontrolled microwave whispering-gallery-mode quasi-optical resonators at 50W LED array illumination, AIP Adv. 5: 087144, 2015.

[2] V. Yurchenko, M. Ciydem, M. Gradziel, A. Murphy, A. Altintas, Light-controlled photonics-based mm-wave beam switch, Opt. Express 24: 15: 16471, 2016.
[3] M. Kunst, G. Beck, The study of charge carrier kinetics in semiconductors by microwave conductivity measurements, J. Appl. Phys., 60: 355-3566, 1986.

[4] G. Beck, M. Kunst, Contactless scanner for photoactive materials using laser-induced microwave absorption, Rev. Sci. Instrum. 57: 197-201, 1988.

[5] D. C. Gupta, F. R. Bacher, W. M. Hughes, Eds., Recombination Lifetime Measurements in Silicon, ASTM special technical publication, 1340: 1-392, 1998.

[6] O. Palais, A. Arcari, Contactless measurement of bulk lifetime and surface recombination velocity in silicon wafers, J. Appl. Phys. 93: 4686-4690, 2003.

[7] K. Lauer, A. Laades, H. Übensee, H. Metzner, A. Lawerenz, Detailed analysis of the microwavedetected photoconductance decay in crystalline silicon, J. Appl. Phys. 104: 104503, 2008.

[8] R.K. Ahrenkiel, S.W. Johnston, Resonant coupling for contactless measurement of carrier lifetime, IEEE 39th PV Specialists Conference, 1389-1393, 2013.

[9] R. K. Ahrenkiel, D. J. Dunlavy, A new lifetime diagnostic system for photovoltaic materials, Sol. Energy Mater. Sol. Cells 95: 1985-1989, 2011.

[10] W. K. Metzger, D. Albin, D. Levi, P. Sheldon, X. Li, B. M. Keyes, and R. K. Ahrenkiel, Time-resolved photoluminescence studies of CdTe solar cells, $J$. Appl. Phys. 94: 5: 3549-3555, 2003.

[11] D. K. Schroder, The concept of generation and recombination lifetimes in semiconductors, IEEE Trans. Electron Devices, 29: 8: 1336-1338, 1982.

[12] A. Barannik, N. Cherpak , A. Kirichenko, Y. Prokopenko, S. Vitusevich, V. Yakovenko, Whispering gallery mode resonators in microwave physics and technologies, Int. J. Microwave Wireless Technolog. 9: 4: 781-796, 2017.

[13] N. W. Chen, J.-W. Shi, H.-J. Tsai, J.-M. Wun, F.-M. Kuo, J. Hesler, T. W. Crowe, J. E. Bowers, Design and demonstration of ultra-fast W-band photonic transmitter-mixer and detectors for $25 \mathrm{Gbits} / \mathrm{sec}$ error-free wireless linking, Opt. Express 20: 19: 21223, 2012.

[14] S.M. Sze, Semiconductor Devices, Physics and Technology, John Wiley and Sons, Hoboken, NJ, 2002.

[15] M.A. Green, M.J. Keevers, Optical properties of intrinsic silicon at $300 \mathrm{~K}$, Prog. Photovoltaics Res. Appl. 3: 3: 189-192, 1995. 